\title{
Modelling the Controlled Release of Toxins in a Rumen Environment ${ }^{+}$
}

\author{
Yue Yuan 1, Emilie Gauthier 1, Natasha L. Hungerford 2, Diane Ouwerkerk 2,3, Mary T. Fletcher 2,* \\ and Bronwyn Laycock 1 ,* \\ 1 School of Chemical Engineering, University of Queensland, Brisbane, QLD 4072, Australia; \\ yue.yuan1@uq.edu.au (Y.Y.); emilie.gauthier@uq.edu.au (E.G.) \\ 2 Queensland Alliance for Agriculture and Food Innovation (QAAFI), The University of Queensland, \\ Brisbane, QLD 4108, Australia; n.hungerford@uq.edu.au (N.L.H.); diane.ouwerkerk@daf.qld.gov.au (D.O.) \\ 3 Agri-Science Queensland, Department of Agriculture and Fisheries (DAF), Brisbane, QLD 4102, Australia \\ * Correspondence: mary.fletcher@uq.edu.au (M.T.F.); b.laycock@uq.edu.au (B.L.) \\ + Presented at the third International Tropical Agriculture Conference (TROPAG 2019), Brisbane, Australia, \\ 11-13 November 2019.
}

Published: 12 February 2020

\begin{abstract}
Pimelea poisoning in grazing cattle, also known as St George or Marree Disease, has been a long-time pestilence for the pastoral industry throughout arid regions of inland Australia. The causative species Pimelea (Thymelaeaceae), native to Queensland, New South Wales and South Australia, have been confirmed, with the secondary metabolite simplexin, a daphnane orthoester, being extracted and identified as the principal toxin. Despite the lack of effective prevention or treatment for Pimelea poisoning, naïve calves have previously been demonstrated to develop detoxification capability following prolonged low-dose simplexin intake. A variety of composites are being fabricated by encapsulating Pimelea plant material or a crude extract in biodegradable and biocompatible polyesters, aiming to develop a sustained toxin release mechanism. Studies on screening potential rumen microflora able to decompose simplexin during rumen-fluid fermentation are being conducted simultaneously. In this project, a quantification method for simplexin within these biocomposites was developed and validated utilising solid-phase extraction combined with UHPLC-Q-Orbitrap MS/MS. Reliable simplexin measurement in matrices will allow investigations into the material composition, geometry and rumen microorganism's effects on the controlled release kinetics of simplexin in vitro. The degradation patterns of toxin delivery systems when exposed to simulated rumen environments will also be thoroughly assessed on both microscopic and chemical scales. Mathematical models of the underlying mass transport mechanisms will ultimately be established through approaches ranging from simple empirical correlations to stochastic simulations, which hold the potential to facilitate future design, optimisation, and prediction of other intra-ruminal devices based on biodegradable polymers.
\end{abstract}

Keywords: Pimelea poisoning; biodegradable biocomposite; slow release; modelling 
Author Contributions: Methodology, Y.Y., N.L.H., E.G.; data analysis, Y.Y.; Writing - original draft preparation, Y.Y.; writing - review and editing, Y.Y., N.L.H., E.G., D.O., M.T.F., B.L.; project administration, M.T.F.; funding acquisition, M.T.F.; supervision, M.T.F., B.L. All authors have read and agreed to the published version of the manuscript.

Funding: This research was funded by Meat \& Livestock Australia, grant number B.GBP.0023.

Acknowledgments: The authors acknowledge the support provided by the Translational Polymer Research Group members at the School of Chemical Engineering of the University of Queensland and the Department of Agriculture and Fisheries technical staff at Agri-Science Queensland.

Conflicts of Interest: The authors declare no conflict of interest.

(C) 2020 by the authors. Licensee MDPI, Basel, Switzerland. This article is an open access article distributed under the terms and conditions of the Creative Commons Attribution (CC BY) license (http://creativecommons.org/licenses/by/4.0/). 